\title{
ON BIORTHOGONAL MATRICES ${ }^{1}$
}

\author{
Y. K. WONG
}

Introduction. Consider the basis consisting of a number system $\mathfrak{A}$ of type $D$, two general ranges $\mathfrak{P}^{1}, \mathfrak{B}^{2}$, and two positive hermitian matrices $\epsilon^{1}, \epsilon^{2}$. We introduce two binary relations for pairs of nonmodular matrices. The matrices $\kappa^{12}, \phi^{21}$ are said to be contraceding as to $\epsilon^{1} \epsilon_{\kappa}^{2}, \epsilon^{2}$ in case $\kappa^{12}, \phi^{21}$ are by columns of $\mathfrak{M}\left(\epsilon^{1}\right), \mathfrak{M}\left(\epsilon^{2}\right)$ respectively and such that $J^{2 \kappa} \kappa^{12} \mu^{2}=J^{2} \phi^{* 12} \mu^{2}$ for every $\mu^{2}$ in the set $\mathfrak{M}\left(\epsilon_{\kappa}^{2} \cap \epsilon^{2}\right)$. It is evident that when $\kappa^{12}$ is of type $\mathfrak{M}\left(\epsilon^{1}\right) \bar{M}\left(\epsilon^{2}\right)$, then the contracedence property implies that $J^{2} \kappa^{12} \phi^{21}=\epsilon_{\kappa}^{1}$ but not conversely. The main results are stated in Theorems 2 and 3 . We next consider $\epsilon_{0}^{1}, \epsilon_{1}^{1}$ both idempotent as to $\epsilon^{1}$. Suppose that $\kappa^{12}$ is by columns of $\mathfrak{M}\left(\epsilon_{0}^{1}\right)$ and $\phi^{21}$ is by rows-conjugate of $\mathfrak{M}\left(\epsilon_{1}^{1}\right)$. Take any pair of vectors $\mu^{1}, \nu^{1}$ modular as to $\epsilon_{0}^{1}, \epsilon_{1}^{1}$ respectively such that $J^{1} \kappa^{* 21} \mu^{1}, J^{1} \phi^{21} \nu^{1}$ are in $\mathfrak{M}\left(\epsilon^{2}\right)$. If $J^{1} \bar{\mu}^{1} \nu^{1}$ is equal to the inner product $J^{2}\left(J^{1} \mu^{1} \kappa^{12}, J^{1} \phi^{21} \nu^{1}\right)$, then $\kappa^{12}, \phi^{21}$ are said to be biorthogonal as to $\epsilon^{1} \epsilon_{0}^{1} \epsilon_{1}^{1} \epsilon^{2}$. When $\epsilon_{1}^{1}=\epsilon_{0}^{1}$, then $\kappa^{12}, \phi^{21}$ are said to be biorthogonal as to $\epsilon^{1} \epsilon_{0}^{1} \epsilon^{2}$ in case they are biorthogonal as to $\epsilon^{1} \epsilon_{0}^{1} \epsilon_{0}^{1} \epsilon^{2}$. With proper restrictions imposed upon $\kappa^{12}, \phi^{21}$, we obtain the contracedence property. In a later paper, we shall establish the relations of biorthogonality and a certain mode of interchange of integration processes.

1. Preliminary results. Consider the basis $\mathfrak{A}, \mathfrak{B}^{1}, \mathfrak{P}^{2}, \epsilon^{1}$, and $\kappa^{12}$ which is by columns of $\mathfrak{M}\left(\epsilon^{1}\right)$. E. H. Moore's generalized Fourier processes give $\epsilon_{\kappa}^{2} \equiv J^{1} \kappa^{* 21} \kappa^{12}$ and $\epsilon_{\kappa}^{1} \equiv J^{2 \kappa} \kappa^{12} \kappa^{* 21}$. The spaces $\mathfrak{M}\left(\epsilon_{\kappa}^{1}\right)$ and $\mathfrak{M}\left(\epsilon_{x}^{2}\right)$ are in one-to-one correspondence (denoted by $\leftrightarrow$ ) via the transformations $J^{1} \kappa^{* 21}$ and $J^{2 \kappa} \kappa^{12}$, and the correspondences are orthogonal in the sense that the moduli of the corresponding vectors are preserved. ${ }^{2}$

(A) $)^{3}$ Suppose that $\mathfrak{M}_{1}\left(\epsilon_{\kappa}^{1}\right) \leftrightarrow \mathfrak{M}_{1}\left(\epsilon_{\kappa}^{2}\right)$ and $\mathfrak{M}_{2}\left(\epsilon_{\kappa}^{1}\right) \leftrightarrow \mathfrak{M}_{2}\left(\epsilon_{\kappa}^{2}\right)$ via the transformations $J^{1} \kappa^{* 21}, J^{2 \kappa} \kappa^{12}$. Then $\mathfrak{M}_{1}\left(\epsilon_{\kappa}^{1}\right)$ is a subset of $\mathfrak{M}_{2}\left(\epsilon_{\kappa}^{1}\right)$ if and only if $\mathfrak{M}_{1}\left(\epsilon_{\kappa}^{2}\right)$ is a subset of $\mathfrak{M}_{2}\left(\epsilon_{\kappa}^{2}\right) ; \mathfrak{M}_{1}\left(\epsilon_{\kappa}^{1}\right)$ is linearly $J^{1}$-closed if and only

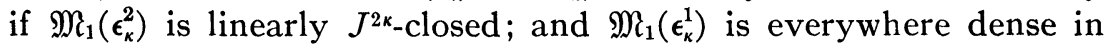
$\mathfrak{M}_{2}\left(\epsilon_{\kappa}^{1}\right)$ if and only if $\mathfrak{M}_{1}\left(\epsilon_{\kappa}^{2}\right)$ is everywhere dense in $\mathfrak{M}_{2}\left(\epsilon_{\kappa}^{2}\right)$.

1 Presented to the Society, June 20, 1940.

${ }^{2}$ For a concise outline of Moore's generalized Fourier theory and its related topics, see Moore, General Analysis, I, pp. 19-26. For an important classical instance, see E. Schmidt, Über die Auflösung linearer Gleichungen mit unendlichvielen Unbekannten, Rendiconti del Circolo Matematico di Palermo, vol. 25 (1908), pp. 56-77.

${ }^{3}$ For the demonstrations of the following results, see the author's forthcoming paper On non-modular matrices. 
Let $\epsilon^{2}$ be a positive hermitian matrix. (B) The class of all vectors $\mu^{1}$ modular as to $\epsilon^{1}$ such that $J^{1} \kappa^{* 21} \mu^{1}$ is in $\mathfrak{M}\left(\epsilon^{2}\right)$ will be denoted by $\mathfrak{M}\left(\epsilon^{1} \kappa^{*} \epsilon^{2}\right)$. The intersection of $\mathfrak{M}\left(\epsilon^{2}\right)$ and $\mathfrak{M}\left(\epsilon_{\kappa}^{2}\right)$ will be denoted by $\mathfrak{M}\left(\epsilon^{2} \cap \epsilon_{\kappa}^{2}\right)$. (C) The set $\mathfrak{M}\left(\epsilon^{2} \cap \epsilon_{\kappa}^{2}\right)$ is identical with the class of vectors $J^{1} \kappa^{* 21} \mu^{1}$ for all $\mu^{1}$ in $\mathfrak{M}\left(\epsilon^{1} \kappa^{*} \epsilon^{2}\right)$. Moreover, (D) the sets $\mathfrak{M}\left(\epsilon_{\kappa}^{1} \kappa^{*} \epsilon^{2}\right)$ and $\mathfrak{M}\left(\epsilon^{2} \cap \epsilon_{\kappa}^{2}\right)$ are in one-to-one (orthogonal) correspondence via the transformations $J^{1} \kappa^{* 21}$ and $J^{2 \kappa} \kappa^{12}$. (E) The set $\mathfrak{M}\left(\epsilon^{1} \kappa^{*} \epsilon^{2}\right)$ is the linear extension of the sum of $\mathfrak{M}\left(\epsilon_{\kappa}^{1} \kappa^{*} \epsilon^{2}\right)$ and the orthogonal complement within $\mathfrak{M}\left(\epsilon^{1}\right)$ of $\mathfrak{M}\left(\epsilon_{\kappa}^{1}\right)$. (F) The class $\mathfrak{M}\left(\epsilon^{1} \kappa^{*} \epsilon^{2}\right)$ is everywhere dense in $\mathfrak{M}\left(\epsilon^{1}\right)$ if and only if $\mathfrak{M}\left(\epsilon^{2} \cap \epsilon_{\kappa}^{2}\right)$ is everywhere dense in $\mathfrak{M}\left(\epsilon_{\kappa}^{2}\right)$.

2. Contraceding pairs of matrices. The basis of the paper consists of a number system $\mathfrak{A}$ of type $D$, two general ranges $\mathfrak{P}^{1}, \mathfrak{P}^{2}$, and two positive hermitian matrices $\epsilon^{1}, \epsilon^{2}$.

Lemma 1. Suppose that $\kappa^{12}$ is by columns of $\mathfrak{M}\left(\epsilon^{1}\right)$. Let $\mathfrak{M}_{0}\left(\epsilon^{1}\right)$ be a subset of $\mathfrak{M}\left(\epsilon^{1}\right)$; then $\mathfrak{M}\left(\epsilon_{\kappa}^{1} \kappa^{*} \epsilon^{2}\right)$ is contained in (contains) $\mathfrak{M}_{0}\left(\epsilon^{1}\right)$ only if $\mathfrak{M}\left(\epsilon^{2} \cap \epsilon_{\kappa}^{2}\right)$ is contained in (contains) the class of vectors $J^{1} \kappa^{* 21} \mu^{1}$ for all $\mu^{1}$ in $\mathfrak{M}_{0}\left(\epsilon^{1}\right)$. The converses are valid provided that $\mathfrak{M}_{0}\left(\epsilon^{1}\right) \subset \mathfrak{M}\left(\epsilon_{\kappa}^{1}\right)$.

Proof. The lemma follows from (D) and (A) in $\$ 1$.

If $\phi^{21}$ is by columns of $\mathfrak{M}\left(\epsilon^{2}\right)$, we introduce the notations due to E. H. Moore:

$$
\stackrel{1}{\epsilon_{\phi}} \equiv J_{\phi}^{2}{ }^{* 12} \phi^{21}, \quad \stackrel{2}{\epsilon_{\phi}} \equiv J_{\phi}^{1 \phi}{ }^{21}{ }^{* 12} .
$$

Lemma 2. Suppose that $\kappa^{12}, \phi^{21}$ are by columns of $\mathfrak{M}\left(\epsilon^{1}\right), \mathfrak{M}\left(\epsilon^{2}\right)$ respectively. Then $\mathfrak{M}\left(\epsilon_{\kappa}^{1} \kappa^{*} \epsilon^{2}\right)$ is contained in (contains) $\mathfrak{M}\left(\epsilon_{\kappa}^{1} \cap \epsilon_{\phi}^{1}\right)$ if and only if $\mathfrak{M}\left(\epsilon_{\kappa}^{2} \cap \epsilon^{2}\right)$ is contained in (contains) the class of vectors $J^{1} \kappa^{* 21} \mu^{1}$ for all $\mu^{1}$ in $\mathfrak{M}\left(\epsilon_{\kappa}^{1} \cap \epsilon_{\phi}^{1}\right)$.

Proof. This lemma is a special instance of Lemma 1.

DEFINITION 1. The matrices $\kappa^{12}, \phi^{21}$ are said to be contraceding as to $\epsilon^{1} \epsilon_{\kappa}^{2} \epsilon^{2}$ in case $\kappa^{12}, \phi^{21}$ are by columns of $\mathfrak{M}\left(\epsilon^{1}\right), \mathfrak{M}\left(\epsilon^{2}\right)$ respectively and such that $J^{2 \kappa} \kappa^{12} \mu^{2}=J^{2} \phi^{* 12} \mu^{2}$ for every $\mu^{2}$ in the set $\mathfrak{M}\left(\epsilon_{\kappa}^{2} \cap \epsilon^{2}\right)$.

Theorem 1. Suppose that $\kappa^{12}, \phi^{21}$ are contraceding as to $\epsilon^{1} \epsilon_{\kappa}^{2} \epsilon^{2}$. Then

(1) $\mathfrak{M}\left(\epsilon_{\kappa}^{1} \kappa^{*} \epsilon^{2}\right)=\left[J^{2} \phi^{* 12} \mu^{2} \mid \mu^{2}\right.$ in $\left.\mathfrak{M}\left(\epsilon^{2} \cap \epsilon_{\kappa}^{2}\right)\right] \subset \mathfrak{M}\left(\epsilon_{\kappa}^{1} \cap \epsilon_{\phi}^{1}\right)$;

(2) $\mathfrak{M}\left(\epsilon^{2} \cap \epsilon_{\kappa}^{2}\right) \subset \mathfrak{M}\left(\epsilon^{2} \phi \epsilon_{\kappa}^{1}\right)$;

(3) $\mathfrak{M}\left(\epsilon^{1} \kappa^{*} \epsilon^{2}\right) \subset \mathfrak{M}\left(\epsilon^{1} \cap \epsilon_{\phi}^{1}\right)$ if and only if every $\mu^{1}$ for which $J^{1} \epsilon_{\kappa}^{1} \mu^{1}=0^{1}$ is in $\mathfrak{M}\left(\epsilon_{\phi}^{1}\right)$.

Proof. Part (1) is a consequence of the fact that

$$
\mathfrak{M}\left(\stackrel{1 \epsilon_{\kappa} \kappa}{* 2}\right)=\left[J^{2 \kappa} \kappa^{12} \mu^{2} \mid \mu^{2} \text { in } \mathfrak{M}\left(\epsilon^{2} \cap \epsilon_{\kappa}^{2}\right)\right]
$$


By hypothesis, we may replace $J^{2 \kappa} \kappa^{12}$ by $J^{2} \phi^{* 12}$. Since $\phi^{* 12}$ is modular as to $\epsilon_{\phi}^{1} \epsilon^{2}$ and $\mathfrak{M}\left(\epsilon_{\kappa}^{1} \kappa^{*} \epsilon^{2}\right)$ is a subset of $\mathfrak{M}\left(\epsilon_{\kappa}^{1}\right)$, every vector of the form $J^{2} \phi^{* 12} \mu^{2}$ must belong to $\mathfrak{M}\left(\epsilon_{\kappa}^{1} \cap \epsilon_{\phi}^{1}\right)$. This proves (1). Part (2) is obvious from (1). Part (3) follows from (1) and (E).

TheOREM 2. Suppose that $\phi^{21}, \kappa^{12}$ are contraceding as to $\epsilon^{2} \epsilon_{\phi}^{1} \epsilon^{2}$. Then

(1) $O^{1} \mathfrak{M}\left(\epsilon_{\kappa}^{1}\right) \subset \mathfrak{M}\left(\epsilon_{\phi}^{1}\right)$ if and only if $\kappa^{12}$ is complete by columns of $\mathfrak{M}\left(\epsilon^{1}\right)$;

(2) the following four assertions are equivalent: (i) $\mathfrak{M}\left(\epsilon^{1} \kappa^{*} \epsilon^{2}\right) \subset \mathfrak{M}\left(\epsilon_{\phi}^{1}\right)$; (ii) $\kappa^{12}$ is complete by columns of $\mathfrak{M}\left(\epsilon^{1}\right)$ and $\mathfrak{M}\left(\epsilon^{2} \cap \epsilon_{\kappa}^{2}\right) \subset \mathfrak{M}\left(\epsilon_{\phi}^{2} \phi^{*} \epsilon^{1}\right)$; (iii) $\kappa^{12}$ is complete by columns of $\mathfrak{M}\left(\epsilon^{1}\right)$ and $\kappa^{12}, \phi^{21}$ are contraceding as to $\epsilon^{1} \epsilon_{\kappa}^{2} \epsilon^{2}$; (iv) $\mathfrak{M}\left(\epsilon^{1} \kappa^{*} \epsilon^{2}\right) \subset\left[J^{2} \phi^{* 12} \mu^{2} \mid \mu^{2}\right.$ in $\left.\mathfrak{M}\left(\epsilon^{2} \cap \epsilon_{\kappa}^{2}\right)\right]$.

If one of the four conditions in (2) is valid, then $\mathfrak{M}\left(\epsilon^{1} \kappa^{*} \epsilon^{2}\right)=\mathfrak{M}\left(\epsilon^{1} \cap \epsilon_{\phi}^{1}\right)$ and $\mathfrak{M}\left(\epsilon^{2} \cap \epsilon_{\kappa}^{2}\right)=\mathfrak{M}\left(\epsilon_{\phi}^{2} \phi^{*} \epsilon^{1}\right)$.

Proof. If $\kappa^{12}$ is complete by columns of $\mathfrak{M}\left(\epsilon^{1}\right)$, then $O^{1} \mathfrak{M}\left(\epsilon_{\kappa}^{1}\right)=\left[0^{1}\right]$, which is, of course, contained in $\mathfrak{M}\left(\epsilon_{\phi}^{1}\right)$. Conversely, every $\mu^{1}$ satisf ying $J^{1} \bar{\mu}^{1} \kappa^{12}=0^{2}$ is in the orthogonal complement within $\mathfrak{M}\left(\epsilon^{1}\right)$ of $\mathfrak{M}\left(\epsilon_{\kappa}^{1}\right)$, and hence is in $\mathfrak{M}\left(\epsilon_{\phi}^{1}\right)$. Thus $J^{1 \phi} \phi^{21} \mu^{1}=0^{2}$. Since $\phi^{21}$ is complete by rows-conjugate of $\mathfrak{M}\left(\epsilon_{\phi}^{1}\right)$, we have $\mu^{1}=0^{1}$, proving that $\kappa^{12}$ is complete by columns of $\mathfrak{M}\left(\epsilon^{1}\right)$.

To prove (i) implies (ii), we observe by (E) that $\mathfrak{M}\left(\epsilon^{1} \kappa^{*} \epsilon^{2}\right)$ is the linear extension of the sum of $\mathfrak{M}\left(\epsilon_{\kappa}^{1} \kappa^{*} \epsilon^{2}\right)$ and $O^{1} \mathfrak{M}\left(\epsilon_{\kappa}^{1}\right)$. If (i) is valid, then by part (1) just proved, $\kappa^{12}$ is complete by columns of $\mathfrak{M}\left(\epsilon^{1}\right)$. By Lemma 2, the condition that $\mathfrak{M}\left(\epsilon_{\kappa}^{1} \kappa^{*} \epsilon^{2}\right) \subset \mathfrak{M}\left(\epsilon_{\phi}^{1}\right)$ is equivalent to

$$
\mathfrak{M}\left(\epsilon^{2} \cap{\underset{\epsilon}{2}}^{2}\right) \subset\left[J^{1}{ }^{* 21} \mu^{1} \mid \mu^{1} \text { in } \mathfrak{M}\left(\epsilon^{1} \cap{ }_{\epsilon_{\phi}}^{1}\right)\right] .
$$

By hypothesis, we may replace $J^{1} \kappa^{* 21}$ by $J^{1 \phi} \phi^{21}$. Since, by (D),

$$
\left[J^{1 \phi} \phi^{21} \mu^{1} \mid \mu^{1} \text { in } \mathfrak{M}\left(\epsilon^{1} \cap \underset{\epsilon_{\phi}}{1}\right)\right]=\mathfrak{M}\left(\underset{\epsilon_{\phi}}{2} \phi^{*} \epsilon^{1}\right),
$$

we have (i) $\rightarrow$ (ii). To prove (ii) $\rightarrow$ (iii), consider any vector $\mu^{2}$ in $\mathfrak{M}\left(\epsilon^{2} \cap \epsilon_{\kappa}^{2}\right)$. If (ii) is valid, then, by (D) (cf. (b) above), there exists a vector $\mu^{1}$ in $\mathfrak{M}\left(\epsilon^{1} \cap \epsilon_{\phi}^{1}\right)$ such that

$$
\mu^{2}=J^{1 \phi} \phi^{21} \mu^{1}
$$

whence

$$
J_{\phi}^{2}{ }^{* 12} \mu^{2}=J^{2}{ }^{* 12} J^{1 \phi}{ }^{21} \mu^{1}=J^{1 \phi} \epsilon_{\phi} \mu^{1}=\mu^{1} .
$$

Now by hypothesis and (c), we have $\mu^{2}=J^{1} \kappa^{* 21} \mu^{1}$. As $\epsilon_{\kappa}^{1}=\epsilon^{1}$, it follows that

$$
J^{2 \kappa}{ }_{\kappa}^{12} \mu^{2}=J_{\kappa}^{2 \kappa}{ }^{12} J_{\kappa}^{1}{ }^{* 21} \mu^{1}=J^{1} \epsilon_{\kappa} \mu^{1}=\mu^{1},
$$


proving the condition (iii). By Theorem 1, we have (iii) $\rightarrow$ (iv). Since $\phi^{* 12}$ is modular as to $\epsilon_{\phi}^{1} \epsilon^{2}$, we secure (iv) $\rightarrow(i)$.

The final statement follows by Theorem 1.

Theorem 3. The matrices $\phi^{21}, \kappa^{12}$ are contraceding as to $\epsilon^{2} \epsilon_{\phi}^{1} \epsilon^{1}, \phi^{21}$ is complete by columns of $\mathfrak{M}\left(\epsilon^{2}\right)$, and $\mathfrak{M}\left(\epsilon^{1} \kappa^{*} \epsilon^{2}\right) \subset \mathfrak{M}\left(\epsilon_{\phi}^{1}\right)$ if and only if $\kappa^{12}$, $\phi^{21}$ are contraceding as to $\epsilon^{1} \epsilon_{\kappa}^{2} \epsilon^{2}, \kappa^{12}$ is complete by columns of $\mathfrak{M}\left(\epsilon^{1}\right)$, and $\mathfrak{M}\left(\epsilon^{2} \phi^{*} \epsilon^{1}\right) \subset \mathfrak{M}\left(\epsilon_{\kappa}^{2}\right)$.

Proof. Apply Theorems 1 and 2.

3. Biorthogonal matrices. By considering $\mathfrak{M}\left(\epsilon_{\kappa}^{1} \kappa^{*} \epsilon^{2}\right)$ and $\mathfrak{M}\left(\epsilon_{\kappa}^{2} \cap \epsilon^{2}\right)$ as subsets of $\mathfrak{M}\left(\epsilon_{\kappa}^{1}\right)$ and $\mathfrak{M}\left(\epsilon_{\kappa}^{2}\right)$ respectively, we have established the one-to-one correspondence between those two subsets. The correspondences are orthogonal. But the Fourier coefficient function of every vector in $\mathfrak{M}\left(\epsilon_{\kappa}^{1} \kappa^{*} \epsilon^{2}\right)$ is also modular as to $\epsilon^{2}$. This property gives rise to another direction of studying the correspondences between the aforementioned subsets.

Definition 2. Suppose that $\epsilon_{0}^{1}, \epsilon_{1}^{1}$ are idempotent ${ }^{4}$ as to $\epsilon^{1}, \kappa^{12}$ is by columns of $\mathfrak{M}\left(\epsilon_{0}^{1}\right)$, and $\phi^{21}$ is by rows-conjugate of $\mathfrak{M}\left(\epsilon_{1}^{1}\right)$. Then $\kappa^{12}$, $\phi^{21}$ are said to be biorthogonal as to $\epsilon^{1} \epsilon_{0}^{1} \epsilon_{1}^{1} \epsilon^{2}$ in case

$$
J^{1} \bar{\mu}^{1} \nu^{1}=J^{2}\left(J^{1} \bar{\mu}^{1} \kappa^{12}, J^{1} \phi^{21} \nu^{1}\right)
$$

for every pair of vectors $\mu^{1}, \nu^{1}$ in $\mathfrak{M}\left(\epsilon_{0}^{1} \kappa^{*} \epsilon^{2}\right), \mathfrak{M}\left(\epsilon_{1}^{1} \phi \epsilon^{2}\right)$ respectively.

It is obvious that $\kappa^{12}, \phi^{21}$ are biorthogonal as to $\epsilon^{1} \epsilon_{0}^{1} \epsilon_{1}^{1} \epsilon^{2}$ if and only if $\phi^{* 12}, \kappa^{* 21}$ are biorthogonal as to $\epsilon^{1} \epsilon_{1}^{1} \epsilon_{0}^{1} \epsilon^{\varepsilon}$.

Definition 3. Suppose that $\epsilon_{0}^{1}$ is idempotent as to $\epsilon^{1}$ and $\kappa^{12}, \phi^{* 12}$ are by columns of $\mathfrak{M}\left(\epsilon_{0}^{1}\right)$. Then $\kappa^{12}, \phi^{21}$ are said to be biorthogonal as to $\epsilon^{1} \epsilon_{0}^{1} \epsilon^{2}$ if they are biorthogonal as to $\epsilon^{1} \epsilon_{0}^{1} \epsilon_{0}^{1} \epsilon^{2}$.

THEOREM 4. Suppose that $\epsilon_{0}^{1}, \epsilon_{1}^{1}$ are idempotent as to $\epsilon^{1}$, and $\kappa^{12}$, $\phi^{* 12}$ are by columns of $\mathfrak{M}\left(\epsilon_{0}^{1}\right), \mathfrak{M}\left(\epsilon_{1}^{1}\right)$ respectively. Then

(i) $\kappa^{12}, \phi^{21}$ are biorthogonal as to $\epsilon^{1} \epsilon_{\kappa}^{1} \epsilon_{1}^{1} \epsilon^{2}$ if $\kappa^{12}, \phi^{21}$ are biorthogonal as to $\epsilon^{1} \epsilon_{0}^{1} \epsilon_{1}^{1} \epsilon^{2}$, and only if $\kappa^{12}, \phi^{21}$ are biorthogonal as to $\epsilon^{1} \epsilon_{\kappa}^{1} \epsilon_{\phi^{*}}^{1} \epsilon^{2}$;

(ii) $\kappa^{12}, \phi^{21}$ are biorthogonal as to $\epsilon^{1} \epsilon_{\kappa}^{1} \epsilon_{1}^{1} \epsilon^{2}$ if and only if

$$
J^{2}\left(J^{1} \bar{\mu}^{1} \phi^{* 12}, \mu^{2}\right)=J^{2 \kappa}\left(J^{1} \bar{\mu}^{1} \kappa^{12}, \mu^{2}\right)
$$

holds for every $\mu^{1}$ in $\mathfrak{M}\left(\epsilon_{1}^{1} \phi \epsilon^{2}\right)$ and every $\mu^{2}$ in $\mathfrak{M}\left(\epsilon^{2} \cap \epsilon_{\kappa}^{2}\right)$.

(iii) $\kappa^{12}, \phi^{21}$ are biorthogonal as to $\epsilon^{1} \epsilon_{\kappa}^{1} \epsilon_{\phi^{*}} \epsilon^{2}$ if and only if

1. $\epsilon_{0}^{1}$ is idempotent as to $\epsilon^{1}$ in case $\epsilon_{0}^{1}$ is by columns of $\mathfrak{M}\left(\epsilon^{1}\right)$ and $J^{1} \epsilon_{0}^{1 *} \epsilon_{0}^{1}=\epsilon_{0}$. See G.A., I, pp. 23-24. 


$$
J^{2} \bar{\xi}^{2} \eta^{2}=J^{1}\left(J^{2 \phi^{*}} \bar{\xi}^{2} \phi^{21}, J^{2 \kappa} \kappa^{12} \eta^{2}\right)
$$

holds for every $\xi^{2}$ in $\mathfrak{M}\left(\epsilon^{2} \cap \epsilon_{\phi^{*}}^{2}\right)$ and every $\eta^{2}$ in $\mathfrak{M}\left(\epsilon^{2} \cap \epsilon_{\kappa}^{2}\right)$.

Proof. Since $\mathfrak{M}\left(\boldsymbol{\epsilon}_{\kappa}^{1}\right)$ and $\mathfrak{M}\left(\epsilon_{\phi^{*}}^{1}\right)$ are subsets of $\mathfrak{M}\left(\epsilon_{0}^{1}\right)$ and $\mathfrak{M}\left(\epsilon_{1}^{1}\right)$ respectively, we have the conclusion (i).

To prove the necessity in (ii), consider any $\mu^{2}$ in the set $\mathfrak{M}\left(\epsilon^{2} \cap \epsilon_{\kappa}^{2}\right)$ and any vector $\mu^{1}$ in $\mathfrak{M}\left(\epsilon_{1}^{1} \phi \epsilon^{2}\right)$. Theorem (D) shows the existence of a vector $\nu^{2}$ in $\mathfrak{M}\left(\epsilon_{\kappa}^{1} \kappa^{*} \epsilon^{2}\right)$ such that

$$
\nu^{1}=J^{2 \kappa} \kappa^{12} \mu^{2}, \quad \mu^{2}=J^{1} \kappa^{* 21} \nu^{1} .
$$

By using the fact that $\kappa^{12}$ is modular as to $\epsilon^{1} \epsilon_{\kappa}^{2}$, we have

$$
J^{2 \kappa}\left(J^{1} \bar{\mu}^{1} \kappa^{12}, \mu^{2}\right)=J^{1} \bar{\mu}^{1} \nu^{1} .
$$

Equations (a) and (b) show that the condition is necessary. The sufficiency can be proved similarly.

Now observe that $\kappa^{12}, \phi^{21}$ are biorthogonal as to $\epsilon^{1} \epsilon_{\kappa}^{1} \epsilon_{\phi^{*}}^{1} \epsilon^{2}$ if and only if

$$
J^{2}\left(J^{1} \bar{\mu}^{1} \phi^{* 12}, \eta^{2}\right)=J^{1}\left(\bar{\mu}^{1}, J^{2 \kappa} \kappa^{12} \eta^{2}\right)
$$

for every $\mu^{1}$ in $\mathfrak{M}\left(\epsilon_{\phi^{*}}^{1} \phi \epsilon^{2}\right)$ and every $\eta^{2}$ in $\mathfrak{M}\left(\epsilon^{2} \cap \epsilon_{\kappa}^{2}\right)$. The latter condition is obviously satisfied if and only if (4.2) holds, since $\mathfrak{M}\left(\epsilon_{\phi^{*}}^{1} \phi \epsilon^{2}\right)$ and $\mathfrak{M}\left(\epsilon_{\phi^{*}}^{2} \cap \epsilon^{2}\right)$ are in one-to-one correspondence via the transformations $J^{1} \phi^{21}$ and $J^{2 \phi^{*}} \phi^{* 12}$ by (D).

Theorem 5. Suppose that $\epsilon_{0}^{1}, \epsilon_{1}^{1}, \kappa^{12}, \phi^{21}$ have the same properties as in the hypothesis of Theorem 4 , and $\mathfrak{M}\left(\epsilon_{\kappa}^{1}\right)$ contains either $\mathfrak{M}\left(\epsilon_{1}^{1}\right)$ or $\mathfrak{M}\left(\epsilon_{0}^{1}\right)$. Then $\kappa^{12}, \phi^{21}$ are biorthogonal as to $\epsilon^{1} \epsilon_{0}^{1} \epsilon_{1}^{1} \epsilon^{2}$ if and only if $\kappa^{12}, \phi^{21}$ are biorthogonal as to $\epsilon^{1} \epsilon_{\kappa}^{1} \epsilon_{1}^{1} \epsilon^{2}$.

Proof. The condition is necessary by Theorem 4 . If $\mathfrak{M}\left(\epsilon_{\kappa}^{1}\right)$ contains $\mathfrak{M}\left(\epsilon_{0}^{1}\right)$, the condition is obviously sufficient. If $\mathfrak{M}\left(\epsilon_{\kappa}^{1}\right)$ contains $\mathfrak{M}\left(\epsilon_{1}^{1}\right)$, the sufficiency is proved by the following argument: Let $\mu^{1}, \nu^{1}$ be vectors in $\mathfrak{M}\left(\epsilon_{1}^{1} \phi \epsilon^{2}\right), \mathfrak{M}\left(\epsilon_{0}^{1} \kappa^{*} \epsilon^{2}\right)$ respectively. Since $\mu^{1}$ is modular as to $\epsilon_{\kappa}^{1}$ by hypothesis, we have

Also

$$
J^{1} \bar{\mu}^{1} \nu^{1}=J^{1}\left(J^{1} \bar{\mu}^{1} \epsilon_{\kappa}, \nu^{1}\right)=J^{1}\left(\bar{\mu}^{1}, J^{1} \epsilon_{\kappa}^{1} \nu^{1}\right) .
$$

$$
J_{\kappa}^{1}{ }^{* 21} \nu^{1}=J^{1} J^{1} \kappa^{* 21} \epsilon_{\kappa} \nu^{1}=J^{1}\left(\kappa^{* 21}, J^{1} \epsilon_{\kappa}^{1} \nu^{1}\right) .
$$

The vector $J^{1} \epsilon_{\kappa}^{1} \nu^{1}$ is evidently in the set $\mathfrak{M}\left(\epsilon_{\kappa}^{1} \kappa^{*} \epsilon^{2}\right)$.

Corollary 6. Suppose that $\kappa^{12}, \phi^{* 12}$ are by columns of $\mathfrak{M}\left(\epsilon^{1}\right), \mathfrak{M}\left(\epsilon_{\kappa}^{1}\right)$ 
respectively. Then $\kappa^{12}, \phi^{21}$ are biorthogonal as to $\epsilon^{1} \epsilon_{0}^{1} \epsilon_{\kappa}^{1} \epsilon^{2}$ if and only if $\kappa^{12}, \phi^{21}$ are biorthogonal as to $\epsilon^{1} \epsilon_{\kappa}^{1} \epsilon^{2}$.

THEOREM 7. Suppose that $\epsilon_{0}^{1}, \epsilon_{1}^{1}, \kappa^{12}, \phi^{21}$ have the same properties as in the hypothesis of Theorem 4 , and $\mathfrak{M}\left(\epsilon_{\kappa}^{2} \cap \epsilon^{2}\right)$ is everywhere dense in $\mathfrak{M}\left(\epsilon_{\kappa}^{2}\right)$. Then $\kappa^{12}, \phi^{21}$ are biorthogonal as to $\epsilon^{1} \epsilon_{0}^{1} \epsilon_{1}^{1} \epsilon^{2}$ and the orthogonal complement within $\mathfrak{M}\left(\epsilon_{1}^{1}\right)$ of $\mathfrak{M}\left(\epsilon_{\phi^{*}}^{1}\right)$ is a subset of $\mathfrak{M}\left(\epsilon_{0}^{1}\right)$ if and only if $\kappa^{12}$, $\phi^{21}$ are biorthogonal as to $\epsilon^{1} \epsilon_{0}^{1} \epsilon_{\phi^{*}}^{1} \epsilon^{2}$ and $\phi^{21}$ is complete by rows-conjugate of $\mathfrak{M}\left(\epsilon_{1}^{1}\right)$.

Proof. The condition is obviously sufficient. For the necessity, we need to show only that $\phi^{21}$ is complete by rows-conjugate of $\mathfrak{M}\left(\epsilon_{1}^{1}\right)$. Consider any $\xi^{1}$ modular as to $\epsilon_{1}^{1}$ such that $J^{{ }^{1}} \phi^{21} \xi^{1}=J^{1} \phi^{21} \xi^{1}=0^{2}$. Then $\xi^{1}$ belongs to $\mathfrak{M}\left(\epsilon_{1}^{1} \phi \epsilon^{2}\right)$. Hence

$$
J^{1} \bar{\mu}^{1} \xi^{1}=J^{2}\left(J^{1} \bar{\mu}^{1} \kappa^{12}, J^{1} \phi^{21} \xi^{1}\right)=0
$$

for every $\mu^{1}$ belonging to $\mathfrak{M}\left(\epsilon_{0}^{1} \kappa^{*} \epsilon^{2}\right)$. Now if $\mathfrak{M}\left(\epsilon_{\kappa}^{2} \cap \epsilon^{2}\right)$ is everywhere dense in $\mathfrak{M}\left(\epsilon_{\kappa}^{2}\right)$, then $\mathfrak{M}\left(\epsilon_{0}^{1} \kappa^{*} \epsilon^{2}\right)$ is everywhere dense in $\mathfrak{M}\left(\epsilon_{0}^{1}\right)$, or, $O^{1} \mathfrak{M}\left(\epsilon_{0}^{1} \kappa^{*} \epsilon^{2}\right)=O^{1} \mathfrak{M}\left(\epsilon_{0}^{1}\right)$. Since $\xi^{1}$ is in $\mathfrak{M}\left(\epsilon_{0}^{1}\right)$ and also in $O^{1} \mathfrak{M}\left(\epsilon_{0}^{1}\right)$, it follows that $\xi^{1}=0^{1}$.

Theorem 8. Assume that $\kappa^{12}$ is of type $\mathfrak{M}\left(\epsilon^{1}\right) \overline{\mathfrak{M}}\left(\epsilon^{2}\right)$, and $\phi^{21}$ is by rows-conjugate of $\mathfrak{M}\left(\epsilon^{1}\right)$ such that $\kappa^{12}, \phi^{21}$ are biorthogonal as to $\epsilon^{1} \epsilon_{\kappa}^{1} \epsilon_{\phi^{*}}^{1} \epsilon^{2}$. Then

(1) $\phi^{* 12}, \kappa^{* 21}$ are contraceding as to $\epsilon^{1} \epsilon_{\phi^{*}}^{2} \epsilon^{2}$;

(2) $\mathfrak{M}\left(\epsilon^{2} \cap \epsilon_{\phi^{*}}^{2}\right) \subset \mathfrak{M}\left(\epsilon^{2} \kappa \epsilon_{\kappa}^{1}\right)$;

(3) $\mu^{1}=J^{2} \kappa^{12} J^{1} \phi^{21} \mu^{1}$ for every $\mu^{1}$ in $\mathfrak{M}\left(\epsilon_{\phi^{*}}^{1} \phi \epsilon^{2}\right)$;

(4) $\mathfrak{M}\left(\epsilon^{2} \cap \epsilon_{\phi^{*}}^{2}\right) \subset\left[J^{1} \phi^{21} \mu^{1} \mid \mu^{1}\right.$ in $\left.\mathfrak{M}\left(\epsilon_{\kappa}^{1} \cap \epsilon_{\kappa^{*}}^{1}\right)\right]$;

(5) $\mathfrak{M}\left(\epsilon_{\kappa}^{1} \phi \epsilon^{2}\right) \supset \mathfrak{M}\left(\epsilon_{\kappa}^{1} \cap \epsilon_{\kappa^{*}}^{1}\right)$ if and only if $\mathfrak{M}\left(\epsilon^{2} \kappa \epsilon_{\kappa}^{1}\right)$ contains $\left[J^{1} \phi^{21} \mu^{1} \mid \mu^{1}\right.$ in $\left.\mathfrak{M}\left(\epsilon_{\kappa}^{1} \cap \epsilon_{\kappa^{*}}^{1}\right)\right]$.

ProOF. Since $\kappa^{12}$ is by rows-conjugate of $\mathfrak{M}\left(\epsilon^{2} \cap \epsilon_{\kappa}^{2}\right)$, we make use of (4.2) in Theorem 4 and secure

$$
J^{2} \kappa^{12} \mu^{2}=J^{1} \epsilon_{\kappa}^{1} J^{2 \phi^{*}} \phi^{* 12} \mu^{2}=J^{2 \phi^{*} \phi^{* 12} \mu^{2}}
$$

for every $\mu^{2}$ belonging to $\mathfrak{M}\left(\epsilon^{2} \cap \epsilon_{\phi^{*}}^{2}\right)$. This proves (1). Part (2) is an immediate consequence of (1) just proved. For the demonstration of (3), we note that $\epsilon_{\kappa}^{1}$ is by columns of $\mathfrak{M}\left(\epsilon_{\kappa}^{1} \kappa^{*} \epsilon^{2}\right)$ and hence by Definition 2 , we have

$$
J^{1} \epsilon_{\kappa} \eta^{1}=J^{2}\left(J^{1} \epsilon_{\kappa}^{112} \kappa^{12}, J_{\phi}^{1} \eta^{21}\right)=J^{2} \kappa^{12} J_{\phi}^{1}{ }^{21} \eta^{1}
$$

for every $\eta^{1}$ in $\mathfrak{M}\left(\epsilon_{\phi^{*}}^{1} \phi \epsilon^{2}\right)$. Since $\mathfrak{M}\left(\epsilon_{\phi^{*}}^{1}\right)$ is a subset of $\mathfrak{M}\left(\epsilon_{\kappa}^{1}\right)$, we have $J^{1} \epsilon_{\kappa}^{1} \eta^{1}=\eta^{1}$, and hence, part (3). To prove (4), consider any $\mu^{2}$ in 
$\mathfrak{M}\left(\epsilon^{2} \cap \epsilon_{\phi^{*}}^{2}\right)$. Let $\mu^{1}$ be a vector in $\mathfrak{M}\left(\epsilon_{\kappa}^{1} \cap \epsilon_{\kappa^{*}}^{1}\right)$ for which $J^{2 \phi^{*}} \phi^{* 12} \bar{\mu}^{2}=\mu^{1}$. Then by (1)

$$
J^{1} \phi^{21} \mu^{1}=J^{1} \phi^{21} J^{2 \phi^{*}} \phi^{* 12} \mu^{2}=J^{2 \phi^{*}} \epsilon_{\phi^{*} \mu}^{2}=\mu^{2},
$$

whence (4) follows. In part (5), if $\mathfrak{M}\left(\epsilon_{\kappa}^{1} \phi \epsilon^{2}\right) \supset \mathfrak{M}\left(\epsilon_{\kappa}^{1} \cap \epsilon_{\kappa^{*}}^{1}\right)$, then

$$
\left[J^{1} \phi^{21} \mu^{1} \mid \mu^{1} \text { in } \mathfrak{M}\left(\epsilon_{\kappa}^{1} \phi \epsilon^{2}\right)\right] \supset\left[J^{1} \phi^{21} \mu^{1} \mid \mu^{1} \text { in } \mathfrak{M}\left(\epsilon_{\kappa}^{1} \cap \epsilon_{\epsilon^{*}}^{1}\right)\right] .
$$

By (C), the left-hand side is identical with $\mathfrak{M}\left(\epsilon^{2} \cap \epsilon_{\phi^{*}}^{2}\right)$, which by part (2) is a subset of $\mathfrak{M}\left(\epsilon^{2} \kappa \epsilon_{\kappa}^{1}\right)$. The converse is obvious.

TheOREM 9. Let $\epsilon_{0}^{1}$ be idempotent as to $\epsilon^{1}$, and $\kappa^{12}$ be of type $\mathfrak{M}\left(\epsilon_{0}^{1}\right) \overline{\mathfrak{M}}\left(\epsilon^{2}\right)$. Suppose that $\phi^{21}$ is by rows-conjugate of $\mathfrak{M}\left(\epsilon_{0}^{1}\right)$ and $\kappa^{12}$, $\phi^{21}$ are biorthogonal as to $\epsilon^{1} \epsilon_{0}^{1} \epsilon^{2}$. Then we have the following conclusions:

(1) $\phi^{21}$ is complete by rows-conjugate of $\mathfrak{M}\left(\epsilon_{0}^{1}\right)$;

(2) $\mu^{1}=J^{2} \kappa^{12} J^{1} \phi^{21} \mu^{1}$ for every $\mu^{1}$ in $\mathfrak{M}\left(\epsilon_{0}^{1} \phi \epsilon^{2}\right)$;

(3) $\mathfrak{M}\left(\epsilon_{0}^{1} \phi \epsilon^{2}\right)=\left[J^{2} \kappa^{12} \mu^{2} \mid \mu^{2}\right.$ in $\left.\mathfrak{M}\left(\epsilon^{2} \cap \epsilon_{\phi^{*}}^{2}\right)\right] \subset \mathfrak{M}\left(\epsilon_{0}^{1} \cap \epsilon_{\kappa^{*}}^{1}\right)$;

(4) $\mathfrak{M}\left(\epsilon^{2} \cap \epsilon_{\phi^{*}}^{2}\right) \subset \mathfrak{M}\left(\epsilon^{2} \kappa \epsilon_{0}^{1}\right)$;

(5) if $\phi^{21}$ is by columns of $\mathfrak{M}\left(\epsilon^{2}\right)$, then $\epsilon_{0}^{1}=J^{2} \kappa^{12} \phi^{21}$ and $\kappa^{12}$ is complete by columns of $\mathfrak{M}\left(\epsilon_{0}^{1}\right)$;

(6) $\mathfrak{M}\left(\epsilon_{0}^{1} \phi \epsilon^{2}\right) \supset \mathfrak{M}\left(\epsilon_{0}^{1} \cap \epsilon_{\kappa^{*}}^{1}\right)$ if and only if every vector in $\mathfrak{M}\left(\epsilon_{0}^{1} \cap \epsilon_{\kappa^{*}}^{1}\right)$ is expressible in the form $J^{2} \kappa^{12} \mu^{2}$, where $\mu^{2}$ is a vector in $\mathfrak{M}\left(\epsilon^{2} \cap \epsilon_{\phi^{*}}^{2}\right)$.

Proof. Part (1) follows from Theorem 7, for $\mathfrak{M}\left(\epsilon^{2} \cap \epsilon_{\kappa}^{2}\right)$ is everywhere dense in $\mathfrak{M}\left(\epsilon_{\kappa}^{2}\right)$ when $\kappa^{12}$ is of type $\mathfrak{M}\left(\epsilon_{0}^{1}\right) \overline{\mathfrak{M}}\left(\epsilon^{2}\right)$. Part (2) is proved in the same way as part (3) of Theorem 8 with the replacement of $\epsilon_{\kappa}^{1}$ by $\epsilon_{0}^{1}$. By Theorem (C), part (3) is a consequence of (2). Part (4) follows from (3). For part (5), we note that $\epsilon_{0}^{1}$ is by columns of $\mathfrak{M}\left(\epsilon_{0}^{1} \phi \epsilon^{2}\right)$; hence the first conclusion follows from (2) whereas the second follows from (1) and the fact that $\phi^{* 12}, \kappa^{* 21}$ are biorthogonal as to $\epsilon^{1} \epsilon_{0}^{1} \epsilon^{2}$. Part (6) is a consequence of (3).

Theorem 10. Suppose that $\kappa^{12}$ is of type $\mathfrak{M}\left(\epsilon^{1}\right) \overline{\mathfrak{M}}\left(\epsilon^{2}\right), \phi^{21}$ is by rowsconjugate of $\mathfrak{M}\left(\epsilon_{\kappa}^{1}\right)$, and $\kappa^{12}, \phi^{21}$ are biorthogonal as to $\epsilon^{1} \epsilon_{\kappa}^{1} \epsilon^{2}$. Then the following assertions are equivalent:

(1) $\mathfrak{M}\left(\epsilon^{2} \cap \epsilon_{\phi^{*}}^{2}\right) \supset \mathfrak{M}\left(\epsilon^{2} \kappa \epsilon_{\kappa}^{1}\right)$;

(2) $\kappa^{12}$ is complete by rows-conjugate of $\mathfrak{M}\left(\epsilon^{2}\right)$ and $\mathfrak{M}\left(\epsilon_{\kappa}^{1} \phi \epsilon^{2}\right)$ contains $\mathfrak{M}\left(\epsilon_{\kappa}^{1} \cap \epsilon_{\kappa^{*}}^{1}\right)$;

(3) $\kappa^{12}$ is complete by rows-conjugate of $\mathfrak{M}\left(\epsilon^{2}\right)$ and $\mathfrak{M}\left(\epsilon^{2} \kappa \epsilon_{\kappa}^{1}\right)$ contains $\left[J^{1} \phi^{21} \mu^{1} \mid \mu^{1}\right.$ in $\left.\mathfrak{M}\left(\epsilon_{\kappa}^{1} \cap \epsilon_{\kappa^{*}}^{1}\right)\right]$;

(4) $\kappa^{* 21}, \phi^{* 12}$ are contraceding as to $\epsilon^{2} \epsilon_{\kappa^{*}}^{1} \epsilon^{1}$, and $\kappa^{12}$ is complete by rows-conjugate of $\mathfrak{M}\left(\epsilon^{2}\right)$; 
(5) $\left[J^{1} \phi^{21} \mu^{1} \mid \mu^{1}\right.$ in $\left.\mathfrak{M}\left(\epsilon_{\kappa}^{1} \cap \epsilon_{\kappa^{*}}^{1}\right)\right] \supset \mathfrak{M}\left(\epsilon^{2} \kappa \epsilon_{\kappa}^{1}\right)$.

If one of the preceding five conditions is valid, then $\epsilon^{2}=\epsilon_{\kappa^{*}}^{2}=J^{1} \phi^{21} \kappa^{12}$.

Proof. The equivalence of the second and third conditions follows from (5) of Theorem 8. Conditions (1), (2), (4), and (5) are equivalent because of Theorem 2 , where $\kappa, \phi, \epsilon^{1}, \epsilon^{2}, \epsilon_{\phi}^{1}$ are replaced by $\kappa^{*}, \phi^{*}, \epsilon^{2}, \epsilon_{\kappa}^{1}, \epsilon_{\phi^{*}}^{2}$ respectively. The relation $\epsilon^{2}=J^{1} \phi^{21} \kappa^{12}$ follows from (4), since $\kappa^{12}$ is by columns of $\mathfrak{M}\left(\epsilon_{\kappa}^{1} \cap \epsilon_{\kappa^{*}}^{1}\right)$.

The University of Chicago 